\title{
Control Theoretical Expression of Quantum Systems And Lower Bound of Finite Horizon Quantum Algorithms
}

\author{
Masahiro Yanagisawa
}

\begin{abstract}
We provide a control theoretical method for a computational lower bound of quantum algorithms based on quantum walks of a finite time horizon. It is shown that given a quantum network, there exists a control theoretical expression of the quantum system and the transition probability of the quantum walk is related to a norm of the associated transfer function.
\end{abstract}

\section{INTRODUCTION}

Quantum analogues of discrete and continuous time random walks have been developed for quantum computation [1], communication [2] and information process [3] as many classical algorithms are based on random walks. Quantum walks provide new possibilities of quantum information engineering such as algorithmic speedup for a specific oracular problem [4], [5], quantum state transmission [6] and entanglement generation over quantum networks [7], [8], [9], [10]. The performance of random walks is evaluated by a probability to reach a certain vertex of a network. Unlike classical random walks, quantum ones do not have limiting there is no limiting distribution due to the unitary evolution, so the performance evaluation is based on averaged distributions. For relatively simple networks, the averaged probability can be obtained for an infinite time interval and an exponential separation is found between the classical and quantum times to propagate through the networks. In reality, however, we are interested in the performance of quantum algorithms for a finite time horizon and generally it is difficult to calculate the averaged distribution because it depends on the time interval.

We give a control theoretical method to find a computational lower bound of quantum algorithms based on quantum walks on any type of networks for the finite time horizon. We first formulate quantum dynamics in a control theoretical way. Given the structure of quantum networks and a Hamiltonian on it, there always exists a transfer function corresponding to the quantum walk due to the linearity of Schrödinger equation. Then the averaged probability is associated with the norm of the transfer function, which is computationally easy to calculate. The only difficulty in this formulation is that the transfer function has poles on the imaginary axis because the evolution of the system is described by a unitary operator. We approximate a given quantum system to a stable system and give the lower bound of the norm of the transfer function.

M. Yanagisawa is with Control and Dyanmical Systems, California Institute of Technology, Pasadena, CA 91125, USA yanagiecds.caltech.edu

\section{Classical and Quantum Walks}

We first describe classical continuous random walks briefly and introduce quantum walks.

\section{A. Classical Random Walk}

A continuous time classical random walk on a graph is a Markov process defined by a set of vertices $\{1, \cdots, l\}$ and a set of edges that specifies which pairs of vertices are connected. Let $\beta$ be a jumping rate. Then the infinitesimal generator is given by

$$
M_{i j}= \begin{cases}-\beta & i \neq j, i \text { and } j \text { connected } \\ 0 & i \neq j, i \text { and } j \text { not connected } \\ c \beta & i=j, c \text { is the valence of vertex } i\end{cases}
$$

The evolution of a probability density vector $\boldsymbol{p}$ is given by

$$
\frac{d \boldsymbol{p}}{d t}=-M \boldsymbol{p},
$$

or equivalently,

$$
\frac{d}{d t} p_{i}=-\sum_{j} M_{i j} p_{j}
$$

where the $i$ th element $p_{i}$ represents the probability of being at the $i$ th vertex. The probability conserves in a sense that $\sum_{i} p_{i}=1$.

The performance of classical algorithms based on random walks is evaluated by a transition probability from a vertex $b$ to a specific vertex $c$ after a long time. Thus, we are usually interested in the limiting distribution

$$
\lim _{t \rightarrow \infty} p_{c}(t),
$$

subject to $p_{b}(0)=1$.

\section{B. Quantum Walk}

A quantum system is described by a complex Hilbert space $\mathcal{H}$. Denote by $\{|1\rangle, \cdots,|l\rangle\}$ the basis of $\mathcal{H}$. (Following the conventional notation of quantum theory, denoted by $|i\rangle$ is the $i$ th normalized basis vector. For example, $|i\rangle$ is a vector whose $i$ th element is 1.) The state of the system is represented by a normalized vector $|\phi\rangle$. The $i$ th element of the vector $|\phi\rangle$ is given by $\langle i \mid \phi\rangle$, where $\langle i|$ is the complex conjugate of $|i\rangle$. This explicit vector expression of the state $|\phi\rangle$ with respect to the basis $\{|i\rangle\},\langle i \mid \phi\rangle$, is sometimes called a probability amplitude. As implied by the name, the vector element $\langle i \mid \phi\rangle$ is related to a probability for the system to be in a state $|i\rangle$, i.e., the probability of being in a state $|i\rangle$ is given by square of the $i$ th vector element $|\phi\rangle,|\langle i \mid \phi\rangle|^{2}$. The 
probability conservation for the quantum system is expressed as $\sum_{i}|\langle i \mid \phi\rangle|^{2}=1$.

The dynamics of the system are described by a Hamiltonian $H$ which is a linear operator in the Hilbert space. In the quantum case, the evolution of the vector is given by the Schrödinger equation

$$
\frac{d}{d t}|\phi\rangle=-\mathrm{i} H|\phi\rangle
$$

or more explicitly, the probability amplitude $\langle i \mid \phi\rangle$ satisfies the wave equation

$$
\frac{d}{d t}\langle i \mid \phi\rangle=-\mathrm{i} \sum_{j}\langle i|H| j\rangle\langle j \mid \phi\rangle,
$$

where we have used the completeness of the basis $\sum_{j}|j\rangle\langle j|=I .\langle i|H| j\rangle$ represents the $(i, j)$ matrix element of the Hamiltonian $H$.

If the system describes a quantum network, the $i$ th basis $|i\rangle$ is identified as the $i$ th vertex of the corresponding graph and the Hamiltonian expresses which pairs of vertices are connected in such a way that

$$
\langle i|H| j\rangle \neq 0 \quad \text { if } i \text { and } j \text { are connected. }
$$

Then, the probability of being at the $i$ th vertex is given by square of the $i$ th element of the vector $|\phi\rangle$, i.e., $|\langle i \mid \phi\rangle|^{2}$. As stated earlier, the probability conservation holds in a sense that $\sum_{i}|\langle i \mid \phi\rangle|^{2}=1$.

An alternative expression of the system is helpful to understand a relation between quantum dynamics and control theory. Assume that the system is initially at the $i$ th vertex $|i\rangle$. After time $t$ the state of the system is expressed as

$$
e^{-\mathrm{i} H t}|i\rangle
$$

A probability that the system is in another vertex $|j\rangle$ after time $t$ is given by

$$
\left|\left\langle j\left|e^{-\mathrm{i} H t}\right| i\right\rangle\right|^{2}
$$

The $(j, i)$ matrix element of $e^{-\mathrm{i} H t}$ is related to the transition probability from $|i\rangle$ to $|j\rangle$. This also characterizes the behavior of the system since an arbitrary initial state can be expanded by the basis $\{|i\rangle\}$. In other words, (9) is a Green function, or a transfer function in terms of control theory.

Unlike the classical case, the limiting distribution (4) does not exist in the quantum case because the evolution is described by the unitary operator. Instead of the limiting distribution, we evaluate the performance of quantum algorithms with a mean distribution defined as

$$
P_{b \rightarrow c}(T):=\frac{1}{T} \int_{0}^{T}\left|\left\langle c\left|e^{-\mathrm{i} H t}\right| b\right\rangle\right|^{2} d t,
$$

which is the transition probability from an initial state $|b\rangle$ to a target state $|c\rangle$ after a time chosen uniformly in $[0, T]$.

\section{TRANSFER FUNCTION AND NORM}

In this section, we give a control theoretical formulation of quantum networks and a relation between the transition probability and the norm of a transfer function.

Let us consider a single input and single output linear stochastic system

$$
\begin{aligned}
d x & =A x d t+b d w, \\
y & =c x .
\end{aligned}
$$

with the initial condition $x(0)=0$. Here we identify the column vector $b$ with $b_{i}=\langle i \mid b\rangle$, i.e., $b$ is the vector expression of $|b\rangle$ from which we start the quantum walk. Likewise, the row vector $c$ is identified with $c_{i}=\langle c \mid i\rangle=$ $\overline{\langle i \mid c\rangle}$ which is the target state of the random walk. $A$ is a skew-Hermitian matrix defined as $A_{i j}=-\mathrm{i}\langle i|H| j\rangle$. $w$ is a Wiener process hypothetically introduced to deal with the quantum system in a control theoretical way, so it has nothing to do with a real physical process. Likewise, the 'state' vector $x$ is an information carrier from the input to the output and independent of the state of the quantum system.

The transfer function of the system is defined as

$$
G(s)=c(s-A)^{-1} b .
$$

Due to the linearity of the Schrödinger equation, there always exists a finite/infinite dimensional linear system (11). Then, the probability of finding the target vertex after the randomly chosen time $t \in[0, T]$ is related to the variance of the output $y$. It follows from the Itô lemma that

$$
P_{b \rightarrow c}(T)=\frac{1}{T} \mathrm{E}[y(T) \overline{y(T)}],
$$

where $\mathrm{E}$ denotes the expectation.

For $T \rightarrow \infty$, the transition probability is formally rewritten as

$$
\begin{aligned}
P_{b \rightarrow c}(T) & =\int_{0}^{T} \frac{d t}{T} \int d \omega e^{\mathrm{i} \omega t} G(\mathrm{i} \omega) \int d \omega^{\prime} e^{\mathrm{i} \omega^{\prime} t} G\left(-\mathrm{i} \omega^{\prime}\right) \\
& =\int d \omega d \omega^{\prime} G(\mathrm{i} \omega) G\left(-\mathrm{i} \omega^{\prime}\right) \int_{0}^{T} \frac{d t}{T} e^{\mathrm{i}\left(\omega-\omega^{\prime}\right) t} \\
& \sim \int d \omega G(\mathrm{i} \omega) G(-\mathrm{i} \omega) \\
& =\|G\|_{2}^{2}
\end{aligned}
$$

where we have used an identity

$$
\lim _{T \rightarrow \infty} \int_{0}^{T} \frac{d t}{T} e^{i\left(\omega-\omega^{\prime}\right) t}=\delta\left(\omega-\omega^{\prime}\right) .
$$

It should be noted that although (15) is widely used in physics, it is not a correct definition of the delta function [11]. In fact, (14) is obviously incorrect because the left hand side of (14) does not satisfy the conditions of norm and the right hand side can diverge. However, this incorrect relation indicates the possibility that the transition probablity $P_{b \rightarrow c}(T)$ is related to a norm of the transfer function $G$. We consider this problem in detail in the next section. 


\section{A LOWER BOUND}

The relation given in the previous section is not satisfactory in both performance and accuracy. Firstly, the right hand side of (14) is not well-defined for quantum networks. Secondly, we need to calculate the transition probability for a long, but finite, time interval. Since our purpose is usually to evaluate how efficient quantum algorithms based on quantum walks are, we are interested in a lower bound of the transition probability for an arbitrary time interval. In this section, we give a rigorous relation between the transition probability and a norm of the transfer function by modifying the linear dynamical system.

There is a significant difference between standard control systems and the system (11). Since the evolution of quantum systems are described by a unitary operator, the matrix $A$ in (11) is skew-Hermitian and the poles of the system are on the imaginary axis of the complex plane. Thus, the transition probability should be related to the power of the output of the system (11). But again, it is not easy to calcualte the power for quantum walks on large networks for an arbitrary time interval. To avoid these difficilties, we introduce a modified transfer function which is stable and close to the original linear system (11).

Due to the modification of the system, the transition probability is not related to a norm of the transfer function by an equality. Here, however, we are particularly interested in a lower bound of the transition probability as stated earlier.

Theorem 1: For a given $\epsilon>0$, let us define a modified transfer function as

$$
G_{\epsilon}(s)=c(s+\epsilon-A)^{-1} b,
$$

For any $T>0$, the transition probability is bounded below as

$$
P_{b \rightarrow c}(T) \geq \frac{1-e^{-2 \epsilon T}}{2 \pi T}\left\|G_{\epsilon}(s)\right\|_{2}^{2} .
$$

Proof: Let $g$ and $g_{\epsilon}$ be the time domain expressions of the transfer functions $G$ and $G_{\epsilon}$, respectively. Denote by $\|g\|_{2,[0, T]}$ the 2-norm of $g$ for a finite time interval, i.e.,

$$
\|g\|_{2,[0, T]}^{2}=\int_{0}^{T}|g(t)|^{2} d t .
$$

Note that $\|g\|_{2,[0, \infty]}=\|g\|_{2}$. Using an inequality

$$
\begin{aligned}
\int_{T}^{\infty} d t\left|g_{\epsilon}(t)\right|^{2} & =\int_{0}^{\infty} d t\left|g_{\epsilon}(t+T)\right|^{2} \\
& \leq e^{-2 \epsilon T} \int_{0}^{\infty} d t\left|g_{\epsilon}(t)\right|^{2}
\end{aligned}
$$

we have

$$
\begin{aligned}
\|g\|_{2,[0, T]}^{2} & \geq\left\|g_{\epsilon}\right\|_{2,[0, T]}^{2} \\
& =\left\|g_{\epsilon}\right\|_{2,[0, \infty]}^{2}-\left\|g_{\epsilon}\right\|_{2,[T, \infty]}^{2} \\
& \geq\left(1-e^{-2 \epsilon T}\right)\left\|g_{\epsilon}\right\|_{[0, \infty]}^{2} \\
& =\frac{1-e^{-2 \epsilon T}}{2 \pi}\left\|G_{\epsilon}\right\|_{2}^{2} .
\end{aligned}
$$

The assertion is complete.

\section{QUANTUM NETWORKS AND SPIN CHAIN}

A spin $1 / 2$ chain is a relatively simple example of (11) for a specific type of quantum computing. In this section, we introduce spin networks and a quantum walk on a graph.

\section{A. Spin Chain}

We first introduce basic notations for a single spin system. The Hilbert space of a single spin $1 / 2$ is a two dimensional complex linear space $\boldsymbol{C}^{2}$. The two basis of $\boldsymbol{C}^{2}$ correspond to spin up and down states along a certain axis. Usually the two basis $\left[\begin{array}{ll}1 & 0\end{array}\right]^{T}:=|\uparrow\rangle$ and $\left[\begin{array}{ll}0 & 1\end{array}\right]^{T}:=|\downarrow\rangle$ are chosen to represent the spin up and down states along $z$-axis, respectively. In this basis, the spin operators are given by

$$
\begin{aligned}
\sigma^{x} & =\left[\begin{array}{ll}
0 & 1 \\
1 & 0
\end{array}\right], \\
\sigma^{y} & =\left[\begin{array}{cc}
0 & \mathrm{i} \\
-\mathrm{i} & 0
\end{array}\right], \\
\sigma^{z} & =\left[\begin{array}{cc}
1 & 0 \\
0 & -1
\end{array}\right] .
\end{aligned}
$$

One can see that $\sigma^{z}|\uparrow\rangle=|\uparrow\rangle$ and $\sigma^{z}|\downarrow\rangle=-|\downarrow\rangle$. These relations mean that the two basis represents the spin up and down states along $z$-axis. Likewise, the two eigenstates of $\sigma^{x}\left(\sigma^{y}\right)$ represents the spin up and down states along $x(y)$ axis, respectively. Note that these operators satisfy a relation

$$
\left\{\sigma_{i}, \sigma_{j}\right\}:=\sigma_{i} \sigma_{j}+\sigma_{j} \sigma_{i}=2 \delta_{i j},
$$

which represents a well-known antisymmetric behavior of spin $1 / 2$ particles.

Let $F=\left\{V_{F}, E_{F}\right\}$ be a graph with the set of vertices $V_{F}$ and edges $E_{F}$. Two vertices $i, j$ are adjacent if $(i, j) \in E_{F}$. We associate a spin system with a graph $F$ by attaching a spin $1 / 2$ to each vertex. The system is then described by a Hilbert space $\mathcal{H}=\left[\boldsymbol{C}^{2}\right]^{\otimes\left|V_{F}\right|}$.

As an example of the graph, we consider an $N$-spin chain with nearest neighbor interactions, as shown in Fig. 1. The Hamiltonian is written as

$$
H^{s}=\sum_{i=0}^{N} \frac{\beta}{2}\left(\sigma_{i}^{x} \otimes \sigma_{i+1}^{y}-\sigma_{i}^{y} \otimes \sigma_{i+1}^{x}\right)
$$

where $\sigma_{i}^{x}, \sigma_{i}^{y}$ and $\sigma_{i}^{z}$ denote the spin operators on the $i$-th vertex of the graph and $\beta$ is the interaction constant between the adjacent spins. Note that the $z$-component of the total spin operator $\sigma^{Z}=\sum_{i \in V_{G}} \sigma_{i}^{z}$ is invariant subject to $H^{s}$ since $\left[\sigma^{Z}, H^{s}\right]=0$. As a result, the Hilbert space $\mathcal{H}$ is decomposed into the direct sum of eigen spaces of $\sigma^{Z}$, i.e., $\mathcal{H}=\bigoplus_{l=0}^{N} \mathcal{H}_{l}$, where $\mathcal{H}_{l}$ is a Hilbert space of $l$ spins up along $z$-axis. Suppose that the system is initially in $\mathcal{H}_{1}$, i.e., only one spin is up and the rest is down. Then the system holds this property for any time subject to the Hamiltonian $H^{s}$, and hence it is described by the natural basis

$$
\Pi_{N}:=\{|0\rangle, \cdots,|N\rangle\},
$$

where a unit vector $|k\rangle$ corresponds to the $k$ th vertex at which a spin is up. 


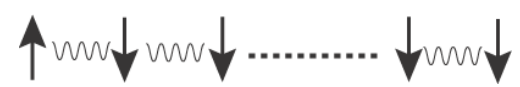

Fig. 1. A spin chain with nearest neighbor interactions. Initially only one spin (at one end of the chain) is up and the rest is down. The total spin conserves under the Hamiltonian (23).

Introducing operators defined as

$$
a_{l}=\frac{1}{2} \prod_{i=0}^{l-1} \sigma_{i}^{z} \otimes\left(\sigma_{l}^{x}-\mathrm{i} \sigma_{l}^{y}\right),
$$

we can rewrite the Hamiltonian (23) as

$$
\begin{aligned}
H^{s} & =\mathrm{i} \beta \sum_{l=0}^{N}\left(a_{l+1} a_{l}^{\dagger}-a_{l} a_{l+1}^{\dagger}\right) \\
& =\mathrm{i} \boldsymbol{a} A^{s} \boldsymbol{a}^{\dagger},
\end{aligned}
$$

where $\boldsymbol{a}:=\left[a_{0} \cdots a_{N}\right]$ and

$$
A^{s}=\beta\left[\begin{array}{ccccc}
0 & 1 & 0 & \cdots & \\
-1 & 0 & 1 & & \\
0 & -1 & 0 & & \\
\vdots & & & \ddots & 1 \\
& & & -1 & 0
\end{array}\right] \text {. }
$$

The matrix expression of Hamiltonian $H^{s}$ with respect to the basis $\Pi_{N}$ is equivalent to $\mathrm{i} A^{s}$ of (11) on the subspace $\mathcal{H}_{1}$.

If we wish to calculate the transition probability from the $i$ th vertex of the spin chain to the $j$ th one, the vectors $b$ and $c$ of (11) are identified with $|i\rangle$ and $|j\rangle$ of the basis (24), respectively. More explicitly, if $i$ is one end of the spin chain and $j$ is the other end, the system is written as

$$
\begin{gathered}
d x=A^{s} x d t+\left[\begin{array}{c}
1 \\
0 \\
\vdots
\end{array}\right] d w, \\
y=\left[\begin{array}{lll}
\cdots & 0 & 1
\end{array}\right] x .
\end{gathered}
$$

with the initial condition $x(0)=0$.

It is worth noting that one can obtain the similar expression for the bosonic systems [10].

\section{B. Quantum Walk on A Graph}

The spin chain introduced above has a similar structure to another type of quantum networks. Let us consider a sequence of graphs consisting of two balanced binary trees of depth $N / 2$ as in Fig.2, and the transition probability from the root of one tree to the other root [4]. The number of vertices, $n$, is of order $2^{N}$. Let $\left\{\left|a_{k}\right\rangle \mid k=1, \cdots, n\right\}$ be vectors representing the vertices. This also constitutes the basis of the Hilbert space. As defined earlier, the Hamiltonian has nonzero matrix element if two vertices $\left|a_{k}\right\rangle$ and $\left|a_{l}\right\rangle$ are connected, i.e., a quantum walk on this graph is defined by a Hamiltonian $\left\langle a_{k}\left|H^{r}\right| a_{l}\right\rangle=\beta / \sqrt{2}$ if $a_{k}$ and $a_{l}(k \neq l)$ are connected. Basically we can obtain the linear dynamical system (11) from this definition. Here we simplify the system in the same way as the spin chain.

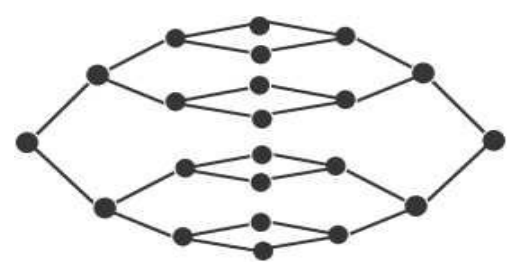

Fig. 2. An example of the binary trees of depth 3 .

We first note that to calculate the transition probability from one root the tree to the other, it is sufficient to know the probability of being $i$ th column (depth), where $i=0, \cdots, N$. Let us define a vector representing the column as

$$
|i\rangle=C \sum_{a \in i \text { th column }}|a\rangle,
$$

where $C$ is a normalization constant. Then, a reduced Hilbert space of $N+1$ dimension is defined by $\{|i\rangle\}$. In this basis, the Hamiltonian is described as

$$
\left\langle i\left|H^{r}\right| i+1\right\rangle= \begin{cases}\beta & i \neq \frac{N}{2} \\ \sqrt{2} \beta & i=\frac{N}{2} .\end{cases}
$$

Thus, for this quantum network the expression (11) is given as

$$
\begin{gathered}
d x=A^{r} x d t+\left[\begin{array}{c}
1 \\
0 \\
\vdots
\end{array}\right] d w, \\
y=\left[\begin{array}{lll}
\cdots & 0 & 1
\end{array}\right] x
\end{gathered}
$$

where

$$
A^{r}=\mathrm{i} \beta\left[\begin{array}{ccccc}
0 & 1 & 0 & \cdots & \\
1 & 0 & 1 & & \\
0 & 1 & 0 & & \\
\vdots & & & \ddots & 1 \\
& & & 1 & 0
\end{array}\right] .
$$

This is the same form as (27) except for a phase difference. Since the probability is not influenced by the phase, (28) and (31) are substantially equivalent.

\section{LOWER BOUND FOR THE EXAMPLE}

In the case of a classical random walk on the graph defined above, the limiting distribution (4) is independent of the initial state and becomes uniform over all vertices after a long time. Since the total number of vertices is $n \sim \mathcal{O}\left(2^{N}\right)$, the probability of being at the other root is $1 / n$. On the other hand, the quantum walk is described by the reduced Hilbert space whose dimension is $N$, and consequently, one can expect that the transition probability (10) is of order $1 / N$, which is exponentially greater than the classical probability. However, this guess is not obvious for a finite time interval. We use (17) to numerically show this distinctive property of the quantum networks. The result is shown in Fig. 3, from which one can see that the transition probability of quantum random walks on the graph is always greater than $1 / N$. 
To see the property of the quantum random walk on the spin chain in more detail, let us introduce another numerical example in which the interactions between spins are randomly determined. Suppose that the Hamiltonian of the spin chain is given by

$$
H_{2}=\sum_{k=1}^{N-1} \sum_{i=0}^{N} \frac{\beta_{k}}{2}\left(\sigma_{i}^{x} \otimes \sigma_{i+k}^{y}-\sigma_{i}^{y} \otimes \sigma_{i+k}^{x}\right),
$$

where $\beta_{k}$ are randomly chosen from a normal distribution. Note that this system still has some regularities. As in (23), this system can be expressed by a Toeplitz matrix in the basis $\Pi_{N}$ defined by (24). While (23) describes the nearest neighbor interactions, in (33) all spins randomly interact with each other, provided that the coefficients of the interactions between spins placed evenly spaced apart are equal. The lower bound of the transition probability from one end to the other end is shown in Fig. 4(A). One can see that the lower bound is still greater than $1 / N$.

Let us consider a case where the coefficients of the interaction Hamiltonian between spins is completely random, i.e.,

$$
H_{3}=\sum_{k=1}^{N-1} \sum_{i=0}^{N} \frac{\beta_{i k}}{2}\left(\sigma_{i}^{x} \otimes \sigma_{i+k}^{y}-\sigma_{i}^{y} \otimes \sigma_{i+k}^{x}\right)
$$

where $\beta_{i k}$ are randomly chosen from a normal distribution. In this case, the matrix expression of the Hamiltonian in the basis $\Pi_{N}$ is given by a random skew Hermitian matrix. The numerical result of the lower bound (17) is shown in Fig. 4(B). Unlike the previous examples, although the decay rate of the lower bound seems to be of order $\mathcal{O}(1 / N)$, it is not greater than $1 / N$ anymore. This can be explained by the wave nature of quantum random walks.

It can be shown that in the case of the uniform nearest neighbor interactions (23), the probability for each spin to be up is given by cylindrical harmonics and can be thought of as propagation on a discrete line [10]. The mode of the propagation in the spin chain are determined by the strength of the interactions between spins. In the case of the Hamiltonian (23), all interactions between spins are uniform and the probability smoothly propagates along the spin chain.

If the interactions between spins are not uniform, differences in the strength of the interactions can be thought of as potential walls by which the propagation is scattered. As a result, the transition probability decreases. In the case of (33), the system still holds structural regularity so that the transition probability is not significantly influenced by the randomness in the Hamiltonian. In the case of (34), however, the potential walls are completely random and impede the propagation along the spin chain significantly.

\section{CONCLUSION}

We have given a control theoretical formulation of quantum systems and shown that the performance of quantum random walks on a graph can be evaluated by the norm of the corresponding transfer function.

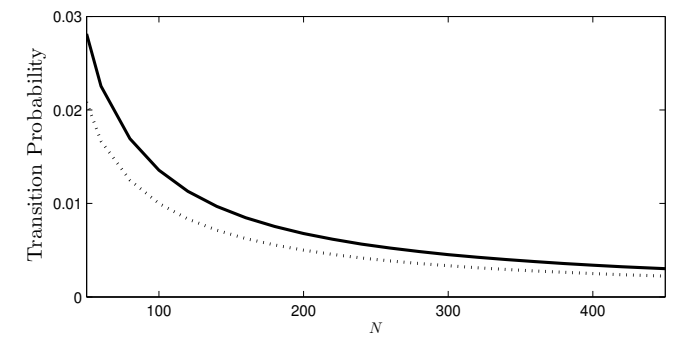

Fig. 3. The transition probability of the spin chain from one end to the other subject to the Hamiltonian (23), or equivalently, the quantum random walk on the graph defined in Sec.V-B (bold line) and $1 / N$ (dotted line).
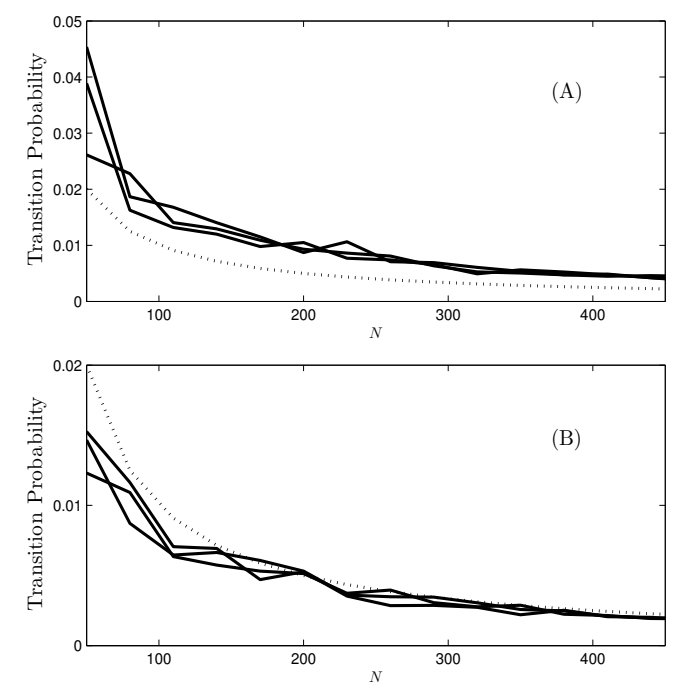

Fig. 4. The transition probability of the spin chain from one end to the other (A) subject to the Hamiltonian (33) (bold line) and (B) subject to the Hamiltonian (34) (bold line, three examples). The dotted lines are $1 / N$.

\section{REFERENCES}

[1] E. Farhi and S. Gutmann, Quantum computation and decision trees, Phys. Rev.A, vol. 58, 915 (1998).

[2] S. Bose, Quantum Communication through an Unmodulated Spin Chain, Phys. Rev. Lett., vol. 91, 207901 (2003).

[3] J. Watrous, Quantum Simulations of Classical Random Walks and Undirected Graph Connectivity, J. Comput. Syst. Sci., vol. 62, 376 (2001).

[4] A. Childs, E. Farhi and S. Gutmann, An Example of the Difference Between Quantum and Classical Random Walks, Quant. Info. Proc., vol 1, 35 (2002).

[5] A.M. Childs, R. Cleve, E. Deotto, E. Farhi, S. Gutmann and D.A. Spielman, Exponential algorithmic speedup by quantum walk quantph/0209131 (2002).

[6] M. Christandl, N. Datta, A. Ekert and A.J. Landahl, Perfect State Transfer in Quantum Spin Networks, Phys. Rev. Lett. vol. 92, 187902 (2004).

[7] L. Amico, A. Osterloh, F. Plastina, R. Fazio and G. M. Palma, Dynamics of entanglement in one-dimensional spin systems, Phys. Rev. A, vol. 69, 022304 (2004).

[8] V. Subrahmanyam, Entanglement dynamics and quantum-state transport in spin chains, Phys. Rev. A, vol. 69, 034304 (2004).

[9] T. J. Osborne and N. Linden, Propagation of quantum information through a spin system, Phys. Rev. A, vol. 69, 052315 (2004).

[10] M. Yanagisawa, Noncommutative optimal control and quantum networks, Phys. Rev. A, vol. 73, 022342 (2006).

[11] I. M. Gel'fand and G. E. Shilov, Generalized functions, Academic Press, New York; 1964. 